\title{
Lean non-alcoholic fatty liver disease: Is there a place for novel antidiabetics in the therapeutic management of this underappreciated "enemy"?
}

\author{
Dimitrios Patoulias ${ }^{1}$ and Michael Doumas ${ }^{1,2}$ \\ ${ }^{1}$ Second Propedeutic Department of Internal Medicine, Aristotle University of Thessaloniki, Thessaloniki, Greece; ${ }^{2}$ Veterans Affairs \\ Medical Center, George Washington University, Washington, D.C, WA, USA
}

Keywords: Non-alcoholic fatty liver disease; Sodium-glucose transporter 2 inhibitors; GLP-1RAs; Obesity; Lean

Dear Editor,

We have read with profound interest the very informative review article authored by Kim and Lee' regarding the effects of antidiabetic drug classes in patients with concomitant non-alcoholic fatty liver disease (NAFLD) and type 2 diabetes mellitus. Therefore, we would like to point out some aspects regarding the phenotype of lean NAFLD, with potential insights into clinical practice.

According to the most recent high level evidence, prevalence of lean NAFLD is $19.2 \%$ within the NAFLD population and $5.1 \%$ within the general population, while it correlates with significant liver and non-liver morbidity and mortality. ${ }^{2}$ Interestingly, besides blood pressure and Homeostatic Model Assessment of Insulin Resistance, lean and obese NAFLD subjects do no differ substantially in terms of physical and metabolic parameters. ${ }^{2}$

Recent real-world data suggest that lean NAFLD subjects might feature greater 15 -year cumulative all-cause mortality and similar cardiovascular and cancer-related mortality, compared to obese
NAFLD patients, ${ }^{3}$ while other have demonstrated that lean NAFLD subjects have lower prevalence of cirrhosis, diabetes, hypertension, dyslipidemia and cardiovascular disease, compared to nonlean subjects with NAFLD. ${ }^{4}$ A sub-analysis of data retrieved from the National Health and Nutrition Survey (NHANES) III database revealed that lean NAFLD patients experience significantly greater all-cause and cardiovascular mortality, compared to lean nonNAFLD subjects. ${ }^{5}$ Despite the high heterogeneity of available data, it is undoubted that lean NAFLD subjects require a thorough initial evaluation and a close monitoring for the development or manifestation of metabolic complications. Of note, another subanalysis of data from the NHANES III database demonstrated that presence of visceral obesity, especially among lean NAFLD patients, has significant prognostic implications regarding all-cause mortality and can be a determinant of selected therapeutic intervention. ${ }^{6}$

Novel antidiabetics, namely glucagon-like peptide-1 receptor agonists (GLP-1RAs) and sodium-glucose co-transporter-2 (SGLT-2) inhibitors have attracted scientific interest during the last decade

\section{Abbreviations:}

GLP-1RAs, glucagon-like peptide-1 receptor agonists; NAFLD, non-alcoholic fatty liver disease; NHANES, the National Health and Nutrition Survey; SGLT-2, sodiumglucose co-transporter-2

\section{Corresponding author: Dimitrios Patoulias}

Second Propedeutic Department of Internal Medicine, Aristotle University of Thessaloniki, Konstantinoupoleos 49, Thessaloniki 54642, Greece

Tel: +30-6946900777, Fax: +30-2310225083

E-mail: dipatoulias@gmail.com

https://orcid.org/0000-0002-6899-684X 
due to their multiple pleiotropic effects, extending far beyond their hypoglycemic effect. Since lean NAFLD patients feature a metabolic profile that does not differ significantly from that observed among obese patients, it seems reasonable that GLP-1RAs and SGLT-2 inhibitors might also have a role in the therapeutic management of these patients, especially when considering their increased cardiovascular risk and the established cardiovascular benefits observed with these drug classes. ${ }^{7}$ Mechanisms of their action are thoroughly discussed by Kim and Lee.'

However, there is an outstanding gap in literature regarding the efficacy and safety of pharmacologic agents, in general, and of novel antidiabetics, in specific, for the management of lean NAFLD, except for some sparse experimental data. ${ }^{8}$ Thus, based on the aforementioned epidemiologic data, there is an urgent need for well-designed prospective studies enrolling lean NAFLD subjects to investigate the efficacy and safety of the promising novel antidiabetics.

\section{Authors' contribution}

Dimitrios Patoulias and Michael Doumas: manuscript writing and critical revision.

\section{Conflicts of Interest}

The authors have no conflict to disclose.

\section{REFERENCES}

1. Kim KS, Lee BW. Beneficial effect of anti-diabetic drugs for nonalcoholic fatty liver disease. Clin Mol Hepatol 2020;26:430-443.

2. Ye Q, Zou B, Yeo YH, Li J, Huang DQ, Wu Y, et al. Global prevalence, incidence, and outcomes of non-obese or lean non-alcoholic fatty liver disease: a systematic review and meta-analysis. Lancet Gastroenterol Hepatol 2020;5:739-752.

3. Zou B, Yeo YH, Nguyen VH, Cheung R, Ingelsson E, Nguyen $\mathbf{M H}$. Prevalence, characteristics and mortality outcomes of obese, nonobese and lean NAFLD in the United States, 1999-2016. J Intern Med 2020;288:139-151.

4. Weinberg EM, Trinh HN, Firpi RJ, Bhamidimarri KR, Klein S, Durlam J, et al. Lean Americans with nonalcoholic fatty liver disease have lower rates of cirrhosis and co-morbid diseases. Clin Gastroenterol Hepatol. 2020 Jul 3. doi: 10.1016/j.cgh.2020.06.066.

5. Golabi P, Paik J, Fukui N, Locklear CT, de Avilla L, Younossi ZM. Patients with lean nonalcoholic fatty liver disease are metabolically abnormal and have a higher risk for mortality. Clin Diabetes 2019:37:65-72

6. Golabi P, Paik JM, Arshad T, Younossi Y, Mishra A, Younossi ZM. Mortality of NAFLD according to the body composition and presence of metabolic abnormalities. Hepatol Commun 2020;4:1136-1148.

7. Zelniker TA, Wiviott SD, Raz I, Im K, Goodrich EL, Furtado RHM, et al. Comparison of the effects of glucagon-like peptide receptor agonists and sodium-glucose cotransporter 2 inhibitors for prevention of major adverse cardiovascular and renal outcomes in type 2 diabetes mellitus. Circulation 2019;139:2022-2031.

8. Ipsen DH, Rolin B, Rakipovski G, Skovsted GF, Madsen A, Kolstrup $S$, et al. Liraglutide decreases hepatic inflammation and injury in advanced lean non-alcoholic steatohepatitis. Basic Clin Pharmacol Toxicol 2018:123:704-713. 